\title{
LETTERS
}

doi:10.1017/S1041610206214418

\section{Visual hallucinations and amnesia associated with zolpidem triggered by fluvoxamine: a possible interaction}

Zolpidem is widely prescribed in clinical practice for the treatment of sleep disorders and it is generally considered safe and well tolerated. However, several cases of hallucinations, amnesia, and delirium associated with the use of zolpidem have been reported previously (Ansseau et al., 1992; Katz, 1995; Toner et al., 2000). We present an elderly woman who experienced visual hallucinations and amnesia associated with zolpidem triggered by fluvoxamine. The patient gave written informed consent to the publication of this letter.

The patient was an 82-year-old Asian woman who came to our outpatient clinic with depressed mood, lassitude, and insomnia. She had not received any prior psychiatric treatment and had no past history of alcohol or substance abuse. After a psychiatric interview, she was diagnosed with a major depressive disorder according to DSM-IV. We prescribed $50 \mathrm{mg} /$ day of fluvoxamine and $10 \mathrm{mg} /$ day of zolpidem, and increased the dose of fluvoxamine to $100 \mathrm{mg} /$ day after two weeks. After one and a half months on this medication, she was admitted to our hospital because her depressive symptoms had not improved. In addition to her depressed mood, lassitude, and insomnia she now also experienced suicidal thoughts. On admission we increased the dose of fluvoxamine from $50 \mathrm{mg}$ two times per day to $50 \mathrm{mg}$ three times per day for her depressive symptoms and maintained the prescribed dose of zolpidem $(10 \mathrm{mg})$ at bedtime. Approximately 30 minutes after taking $10 \mathrm{mg}$ of zolpidem at 2030 on the third day of her admission, she said confusedly, "My grandson comes and sees me. I want to go home with him." She had experienced an episode of visual hallucination, which lasted from several minutes to half an hour almost every night. The next morning she could not remember anything of the previous night. We re-examined a blood test including glucose level, ammonia concentration, and endocrine disturbance, and conducted electroencephalography and magnetic resonance imaging of the brain. These laboratory tests showed that everything was within normal limits there were no abnormal findings that might cause visual hallucinations and amnesia. We postulated that possible fluvoxamine-zolpidem interaction induced her visual hallucinations and amnesia, and so discontinued the zolpidem $10 \mathrm{mg}$ dose at bedtime. Subsequently, her nightly visual hallucinations and amnesia disappeared. After four weeks of taking fluvoxamine $150 \mathrm{mg} /$ day, her depressive symptoms ameliorated and she was discharged and able to go home. Two months 
later, she had no visual hallucinations and amnesia and her depressive symptoms had been in remission.

Toner et al. (2000) described four major variables - zolpidem dose, gender, protein binding affinity, and cytochrome P450 3A4 isoenzyme inhibition which must be considered when prescribing zolpidem concomitantly with antidepressants. Almost all patients who suffered adverse effects, such as hallucinations, amnesia, and delirium associated with the use of zolpidem, had taken doses greater than $10 \mathrm{mg}$ per day (Ansseau et al., 1992; Katz, 1995; Toner et al., 2000). Women are found to have higher serum zolpidem concentrations than men at equivalent dosages (Toner et al., 2000). Zolpidem is mainly metabolized via the cytochrome P450 3A4 isoenzyme (von Moltke et al., 1999). Therefore, the serum zolpidem concentration would be made higher by concomitantly used selective serotonin reuptake inhibitors or other drugs which possessed a degree of cytochrome P450 3A4 isoenzyme inhibition. On the other hand, another case report described a young woman who presented with delirium following intake of zolpidem and paroxetine, which did not inhibit the cytochrome P450 3A4 isoenzyme but possessed cytochrome P450 2D6 isoenzyme inhibition (Katz, 1995). The author noted that since both zolpidem and paroxetine were highly protein bound, they might interact through competitive binding, and this interaction could increase the amount of free zolpidem, which would result in delirium (Katz, 1995). Patients with a low serum albumin concentration, for example those suffering malnutrition, are susceptible to developing hallucinations or delirium with the use of zolpidem. In our patient, the dose of fluvoxamine was increased from $100 \mathrm{mg}$ to 150 $\mathrm{mg} /$ day on admission, and three days later, she experienced an episode of visual hallucinations and amnesia. Her laboratory tests, both on admission and during these episodes, showed no abnormal findings and, in particular, no low serum albumin concentration. Fluvoxamine potently inhibits cytochromes P450 1A2 and $2 \mathrm{C} 19$, and has a mild to moderate inhibitory effect on $2 \mathrm{C} 9,2 \mathrm{D} 6$, and $3 \mathrm{~A} 4$ (Hemeryck and Belpaire, 2002). The plasma protein binding of fluvoxamine is approximately $77 \%$ (Hemeryck and Belpaire, 2002). According to these findings and previous reports, concomitant administration of zolpidem and fluvoxamine is relatively safe and tolerated. In this case, however, there appears to be a temporal causal relationship between an increase in fluvoxamine administered concomitantly with zolpidem and the occurrence of visual hallucinations and amnesia. These symptoms ameliorated when zolpidem was discontinued. We therefore conclude that it is possible that a fluvoxamine-zolpidem interaction induced the visual hallucinations and amnesia.

In conclusion, this is the first case to our knowledge of visual hallucinations and amnesia reported in the literature that may be related to a possible fluvoxamine-zolpidem interaction. Concomitant administration of zolpidem and 
fluvoxamine is relatively safe according to previous findings, but the case reported here suggests that clinicians should consider possible fluvoxamine-zolpidem interaction as a cause of visual hallucinations and amnesia in an elderly woman.

\section{References}

Ansseau, M., Pitchot, W., Hansenne, M. and Gonzalez Moreno, A. (1992). Psychotic reactions to zolpidem. Lancet, 339, 809.

Hemeryck, A. and Belpaire, F. M. (2002). Selective serotonin reuptake inhibitors and cytochrome P-450 mediated drug-drug interactions: an update. Current Drug Metabolism, 3, 13-37.

Katz, S. E. (1995). Possible paroxetine-zolpidem interaction. American fournal of Psychiatry, $152,1689$.

Toner, L. C., Tsambiras, B. M., Catalano, G., Catalano, M. C. and Cooper, D. S. (2000). Central nervous system side effects associated with zolpidem treatment. Clinical Neuropharmacology, 23, 54-58.

von Moltke, L. L. et al. (1999). Zolpidem metabolism in vitro: responsible cytochromes, chemical inhibitors, and in vivo correlations. British fournal of Clinical Pharmacology, 48, 89-97.

\section{SHINSUKe Kito AND YoshiHIKo KogA}

Department of Neuropsychiatry, Kyorin University School of Medicine, Tokyo, Japan

doi:10.1017/S1041610206224414

\section{A practice audit of maintenance electroconvulsive therapy in the elderly}

Maintenance ECT (m-ECT) is a possible alternative treatment for the long-term management of chronic, recurrent, medication-resistant psychiatric disorders. However, the practice of m-ECT is highly variable (Kramer, 1987) with a dearth of research findings or guidelines specific to the elderly. A review of the existing literature found that while most research comprised case studies and small open studies, m-ECT appeared to be a safe and effective treatment that is possibly underused (Andrade and Kurinji, 2002). The gaps in the available evidence base have not assisted in resolving the debate about the most appropriate contemporary use of m-ECT (Scott, 2005). The American Psychiatric Association provides detailed guidelines for the use of m-ECT (APA, 1990), while the National Institute for Clinical Excellence guidelines (U.K. ECT Review Group, 2003) strongly discourage its use, citing the lack of conclusive evidence to support its effectiveness. Such discrepant views highlight the fact that the practice, use and efficacy of m-ECT remain largely unknown in this difficult-to-treat elderly cohort.

A retrospective audit was conducted of patients receiving $\mathrm{m}$-ECT at the St George's Aged Psychiatry Service, Melbourne, Australia from 1997 to 2004 
(inclusive). All patients who had received m-ECT following an acute inpatient course of ECT were identified. The medical files of these patients were obtained and comprehensively audited. Information collected included demographic data, psychiatric and medical diagnoses and medications. The m-ECT record was reviewed and the date, setting (inpatient or outpatient), electrode placement (unilateral or bilateral) and the patient's legal status (voluntary or involuntary) for each treatment were noted. Data were gathered relating to the clinical effect, efficacy and tolerability of m-ECT. Clinical parameters pre- and postcommencement of m-ECT were compared, including the number of psychiatric hospital admissions and length of stay in hospital (or bed days).

Seventeen patients were included in this practice audit. The average age of patients was 82.2 (range $=67-94$ years). Fourteen of the 17 patients were female. Fourteen patients were diagnosed with major depressive disorder, one had bipolar affective disorder and three had primary psychotic disorders (schizophrenia or schizoaffective disorder). The duration of psychiatric illness varied between 1 and 65 years, with 13 patients being chronic sufferers for at least 10 years. Fifteen of the patients audited had active comorbid medical problems requiring treatment at the time of $\mathrm{m}$-ECT.

Of the 13 patients with major depressive disorder, an average of 4.5 antidepressants had been trialled per patient, prior to the commencement of m-ECT. Seven of these 13 had trialled at least one adjunctive mood stabiliser. All seven had received lithium at some stage. Nine of the 13 had trialled an adjunctive antipsychotic.

Of the three patients with a primary psychotic disorder, an average of 7.7 antipsychotic medications had been trialled per patient prior to the commencement of $\mathrm{m}$-ECT. All had received trials of at least one adjunctive mood stabiliser.

The sum total of m-ECT treatments for all 17 patients was 773 for the duration of this audit. The number of m-ECT treatments received by each patient varied from 7 to 189 with an average of 45.5 treatments (median $=23$, $\mathrm{SD}=57.9$ ). The course duration ranged between 3 and 60 months with an average of 22.9 months (median $=13.5, \mathrm{SD}=20.5$ ). The frequency of m-ECT ranged between one treatment every 9.2 days and one treatment every 28.6 days (approximately weekly to monthly). The average inter-treatment interval was 15.3 days (median $=13.2, \mathrm{SD}=6.6$ ). Sixteen of the 17 patients received bilateral ECT. Eight patients received all $\mathrm{m}$-ECT treatments voluntarily while five patients received all $\mathrm{m}$-ECT involuntarily.

Given that the course duration of m-ECT varied significantly for each patient, a comparison was made of clinical outcome measures for an equivalent period of time before and after the commencement of m-ECT. For example, if a patient had received 12 months of $\mathrm{m}-\mathrm{ECT}$, clinical outcomes for the 12 months 
preceding the commencement of m-ECT were compared with the 12 months during which the patient received m-ECT. Thirteen of the 17 patients had a lower number of admissions to hospital following the commencement of m-ECT, with seven patients having no further psychiatric admissions. This difference was statistically significant $(p<0.015)$ using a paired $t$-test. The length of stay in hospital was totalled for the two periods, pre- and post-commencement of mECT (2380 and 1004 bed days) and compared. Fourteen of the 17 patients had a lower number of bed days following the commencement of m-ECT. This difference was again statistically significant $(\mathrm{p}<0.010)$.

Ten of the 17 patients did not have any documented side-effects relating to $\mathrm{m}$ ECT. The most common side-effects recorded included confusion, delirium and short-term memory loss. These were short-lived and improved spontaneously. In addition, files were specifically reviewed for comments relating to the patients' subjective experience of m-ECT treatment. Three of the 17 patients recurrently presented with extreme anxiety preceding $\mathrm{m}-\mathrm{ECT}$, despite relaxation techniques and involvement of families. In total, eight of the 17 patients had a documented strong dislike for this mode of treatment.

Three of the 17 patients were still continuing m-ECT at the conclusion of this audit. Of the remaining 14, reason(s) were documented to explain the cessation of m-ECT including: illness remission (seven patients), no perceived additional benefit to psychotropic medication (five), side-effects (six), refusal to consent (four) and objection from the family (one). All patients continued psychotropic medication after ceasing m-ECT with four patients prescribed combination therapy.

Following discontinuation of m-ECT, six patients remained in illness remission while eight patients showed residual psychiatric symptoms. Three patients clinically relapsed with two requiring further psychiatric admission.

The results of this practice audit indicate that $\mathrm{m}$-ECT is an effective treatment for psychiatrically unwell elderly patients, with a significant majority having fewer hospital admissions and bed days following the commencement of m-ECT. It appears to be a safe and well-tolerated treatment modality in this group despite almost all having active comorbid medical problems.

More research is required in order to better inform our practice of m-ECT in the elderly population.

\section{References}

APA (1990). The Practice of ECT: Recommendations for Practice, Training and Privileging. Task Force Report on ECT. Washington DC: American Psychiatric Press.

Andrade, C. and Kurinji, S. (2002). Continuation and maintenance ECT: a review of recent research. Fournal of ECT, 18, 149-158. 
Kramer, B. A. (1987). Maintenance ECT: a survey of practice (1986). Convulsive Therapy, 3, $260-268$.

Scott, A. I. (2005). College guidelines on electroconvulsive therapy: an update for prescribers. Advanced Psychiatric Treatments, 11, 150-156.

U.K. ECT Review Group (2003) Efficacy and safety of electroconvulsive therapy in depressive disorders: a systematic review and meta-analysis. Lancet, 361, 799-808.

LYN-MAY LIM

Consultant Psychiatrist, St George's Aged Psychiatry Service, Melbourne, Australia

Email: lynmaylim@gmail.com

doi:10.1017/S1041610206234410

\section{Implications of daily, monthly and seasonal variation of new referrals for working patterns of a community mental health team}

Our unit is currently developing a new model of working with the development of two Community Mental Health Teams (CMHTs) following the National Service Framework for Older People (www.dh.gov.uk/policyAndGuidance/ HealthAndSocialCareTopics/OlderPeoplesServices/fs/en) and the New Ways of Working document (www.dh.gov.uk/PolicyAndGuidance/HumanResources AndTraining/NewWaysOfWorking/fs/en). Prior to the commencement of the CMHTs in October 2005, all new referrals were received by the responsible consultant and the subsequent initial assessment was conducted by the Consultant or the Specialist Registrar. However, since the commencement of the CMHTs, all new referrals are received by the CMHT and allocated to the most appropriate CMHT member (including psychiatrists, community psychiatric nurses (CPNs), Social Workers and Psychologists) for the initial assessment. In addition, there is a rostered duty system whereby CMHT members are on duty to process elective referrals and deal with urgent referrals. For some members of the CMHT, like the CPNs, these duties were in addition to the work they were doing prior to the advent of the CMHTs.

Identification of the daily, monthly and seasonal pattern of new referrals was undertaken because variations in the timing of the referrals may allow appropriate planning of the timing of fixed CMHT meetings, educational activities and the availability of an adequate staffing complement. We are unaware of any literature on the timing (daily, monthly and seasonal) of new referrals to Old Age Psychiatry Services. The day, month and season of all referrals made to the two consultant teams that served the same catchment area as one of the CMHTs, for a two-year period (ending 30th September 2005) immediately preceding the commencement of the CMHT, were identified from the referrals book. 
There were 672 new referrals. The daily, monthly and seasonal variation of all referrals are illustrated in Tables S1, S2 and S3 (Tables are published electronically at www.journals.cambridge.org/jid_IPG) respectively. There was a higher rate of referrals on Monday, Tuesday and Wednesday and a lower rate of referral on Thursday and Friday. The referral rate was lower in January, March and April, but higher in July. The referral rate was lower in the Spring, but similar in the other three seasons.

These findings have implications for when non-clinical CMHT activities and elective clinical meetings are organized; for example, non-clinical activities like teaching and training, team-building, away days and management meetings could be organized during time-periods when the anticipated referral rate is low. Also, there are implications for the CMHT management to ensure that an adequate complement of CMHT staff is available during the anticipated periods of higher referral rates. These measures would enable the service to continue to provide a rapidly responsive service and also allow a more even daily, monthly and seasonal distribution of clinical work. These findings also have implications for the controversial issue of planned annual or study leave for the CMHT staff. We hope to repeat this exercise after our CMHT has been up and running for a full year.

\author{
Ajit Shah and Mellisha Padayatchi \\ West London Mental Health NHS Trust \\ Uxbridge Road, Southall, Middlesex \\ UB1 3EU, U.K. \\ E-mail: ajit.shah@wlmht.nhs.uk
}

doi:10.1017/S1041610206244417

\title{
Catechol-0-methyltransferase gene polymorphism in dementia with Lewy bodies-related psychosis: evidence for a genetic predisposition
}

Dementia with Lewy bodies' (DLB) patients commonly experience psychotic symptoms, most notably visual hallucinations, which represent one of the core features of clinical diagnosis. Notwithstanding, their frequency is variably estimated and their pathophysiology is still debated. Literature has recently developed an increased interest in defining genetic correlates of psychiatric symptoms in dementia, suggesting that genes involved in dopamine breakdown might contribute to psychosis risk.

Among others, the cathecol-o-methyltransferase (COMT) gene contains a functional common polymorphism characterized by Valine ( $\mathrm{H}$ allele) to Methionine (L allele) substitution (Akil et al., 2003). The presence of Valine 
in the coding sequence leads to the up-regulation of striatal dopamine activity, thus predisposing to an increased risk to psychosis susceptibility in Alzheimer Disease (AD) (Borroni et al., 2006; Sweet et al., 2005).

No association studies between COMT polymorphism and DLB have been performed so far. The present study aimed to evaluate whether functional COMT genetic variation is a risk factor for DLB-related psychosis.

Seventy-five DLB patients were recruited, and had been followed for at least 1 year after enrollment, and the diagnosis of probable DLB had been made. Each patient underwent a neuropsychological and behavioral evaluation and a structural neuroimaging study. In particular, psychotic symptoms (delusions or hallucinations) were required to be present, at least intermittently, for 1 month or longer, and severe enough to cause some disruption in patients' and/or others' functioning. Their intensity and frequency were scored by Neuropsychiatry Inventory (NPI) scale.

$C O M T$ genotyping was performed blinded to diagnosis, as previously reported (Borroni et al., 2006), and its association with psychosis, which occurred in $53.4 \%$ of the sample $(n=40)$ analyzed.

The presence of at least one ${ }^{*} \mathrm{H}$ allele $\left(C O M T^{*} \mathrm{H}=C O M T \mathrm{~L} / \mathrm{H}\right.$ or $C O M T$ $\mathrm{H} / \mathrm{H})$ was significantly associated with the presence of psychosis $(62.3 \%)$ compared to COMT non ${ }^{*} \mathrm{H}$ carriers $(31.8 \%, P<0.02)$. Delusion and hallucination NPI scores were individually considered, and $C O M T^{*} \mathrm{H}$ genotype was associated with more severe and more frequent presence of both hallucinations $(2.8 \pm 0.5$ vs. $0.9 \pm 0.3, P<0.3)$ and delusions $\left(C O M T^{*} \mathrm{H}\right.$ vs. $C O M T^{*} \mathrm{~L}$, mean \pm standard error, $2.0 \pm 0.4$ vs. $0.3 \pm 0.2, \mathrm{P}<0.2)$. No significant association between COMT genotype and either demographic and clinical characteristics, other NPI items or total NPI was found.

This study suggests that psychosis in DLB might be mediated by genes, $C O M T$, that contribute to psychosis risk across disorders, as already demonstrated in schizophrenia and in AD (Borroni et al., 2006; Sweet et al., 2005). The pathophysiology of the relationship between COMT genotype and psychosis remains still unclear, but it might be argued for a central role of the different regulation of striatal dopamine in $C O M T^{*} \mathrm{H}$ carriers (Akil et al., 2003). Further studies on this issue are warranted to confirm these preliminary findings.

\section{References}

Akil, M., Kolachana, B. S., Rothmond, D. A., Hyde, T. M., Weinberger, D. R.and Kleinman, J. E. (2003). Catechol-O-methyltransferase genotype and dopamine regulation in the human brain. Fournal of Neuroscience, 23, 2008-2013.

Borroni, B. et al. (2006). Cumulative effect of COMT and 5-HTTLPR polymorphisms and their interaction with disease severity and comorbidities on the risk of psychosis in Alzheimer disease. American fournal of Geriatric Psychiarry, 14, 343-351. 
Sweet, R. A. et al. (2005). Catechol-o-methyltransferase haplotypes are associated with psychosis in Alzheimer disease. Molecular Psychiatry, 10, 1026-1036.

\author{
Barbara Borroni, ${ }^{1}$ Monica Di LuCA ${ }^{2}$ AND Alessandro \\ PADOVANI ${ }^{1}$ \\ ${ }^{1}$ Department of Neurology, University of Brescia, Brescia and Centre for Behavioural \\ Disturbances and Neurodegenerative Disease, University of Brescia, Brescia \\ ${ }^{2}$ Department of Pharmacological Sciences, Centre of Excellence for Neurodegenerative \\ Disorders, Milan, Italy \\ E-mail: borroni@med.unibs.it
}

doi:10.1017/\$1041610206254413

\title{
Towards education-fair dementia screening
}

The study by Rowland et al. (2006) has shown that the six-item Rowland Universal Dementia Assessment Scale (RUDAS) is as accurate as the MMSE in identifying dementia. A high positive likelihood ratio of RUDAS makes it useful for ruling-in dementia in cases where the disorder is suspected.

In a similar study from Kerala in South India (Iype et al., 2006), we compared RUDAS and MMSE. Our patients with dementia had only mild to moderate dementia with mean RUDAS and MMSE scores of 14.16 (SD 6.92) and 13.69 (SD 6.74), respectively. Our subjects were comparatively younger with mean ages of 65.1 years for those with dementia and 65.07 years for those in the control group. There was correlation between RUDAS and MMSE scores, with Spearman's correlation coefficient being 0.486 (95\% CI 0.326-0.646, $\mathrm{p}<0.0001$ ). We found that gender has no effect on RUDAS and MMSE scores.

On ROC curve analysis, the area under the curve for RUDAS was 0.931 (95\% CI 0.885-0.976) and that for MMSE was 0.847 (95\% CI $0.776-0.917$. We found that the ideal cut-off for RUDAS was 22 with sensitivity of 0.862 (95\% CI $0.773-0.951)$, specificity of 0.879 (95\% CI 0.795-0.963), positive likelihood ratio of 7.12 and negative likelihood ratio of 0.16 . The ideal cut-off for MMSE was 20 with sensitivity of 0.810 (95\% CI $0.709-0.911)$, specificity of 0.724 (95\% CI 0.609-0.839), positive likelihood ratio 2.94 and negative likelihood ratio of 0.26 . Thus we too found RUDAS effectively ruling in dementia below the ideal cut-off $22 / 30$.

We examined the effect of education on RUDAS scores among our controls. There was positive correlation between RUDAS scores and number of years of formal education (Pearson correlation coefficient $=0.452, \mathrm{p}<0.001$ ) (Iype et al., 2006). Hence our findings do not support the claim of Rowland et al. (2006) that RUDAS is free from educational bias. However, we found RUDAS to have less educational bias in comparison to MMSE. 
People with less education, especially those who are illiterate and innumerate, tend to perform poorly on cognitive assessments. Thus they are likely to be screened positive for dementia even in the absence of cognitive impairment. Hence it is important to use educationally fair tests when screening for dementia. However, complete elimination of educational bias seems to be difficult to achieve. For example, the cognitive assessment section of the Community Screening Instrument for Dementia (CSI-D) (Hall et al., 1993) continues to have educational bias though it has no literacy-dependent items (Prince et al., 2003). At the same time, the scores generated by the informant section of CSI-D are educationally fair. Combining the scores generated by cognitive assessment and the informant report had helped CSI-D to become an education-fair screening instrument (Prince et al., 2003).

We have found RUDAS to be more user-friendly than the cognitive assessment section of CSI-D. We suggest the addition of a brief informant interview to RUDAS for further reduction of educational bias. The items from the informant section of CSI-D (Hall et al., 1993) and Everyday Abilities Scale for India (EASI) (Fillenbaum et al., 1999) may be considered when developing an informant section for RUDAS.

\section{References}

Fillenbaum, G. G. et al. (1999). Development of an activities of daily living scale to screen for dementia in an illiterate rural older population in India. Age and Ageing, 28, 161-168.

Hall, K. S. et al. (1993). The development of a dementia screening interview in two distinct languages. International fournal of Methods in Psychiatric Research, 3, 1-28.

Iype, T., Ajitha, B. K., Antony, P., Ajeeth, N. B., Job, S. and Shaji, K. S. (2006). Usefulness of the Rowland Universal Dementia Assessment Scale in South India. Fournal of Neurology, Neurosurgery and Psychiatry, 77, 513-514.

Prince, M., Acosta, D., Chiu, H., Scazufca, M., and Varghese, M. (2003). Dementia diagnosis in developing countries: A cross-cultural validation study. Lancet, 361, 909-917.

Rowland, J. T., Basic, D., Storey, J. E. and Conforti, D. A. (2006). The Rowland Universal Dementia Assessment Scale (RUDAS) and the Folstein MMSE in a multicultural cohort of elderly persons. International Psychogeriatrics, 18, 111-120.

Thomas Iype, ${ }^{1}$ B. K. AJitha ${ }^{2}$ AND K. S. ShajI ${ }^{2}$

${ }^{1}$ Medical College, Thiruvandapuram, Kerala, India

Email: beenaiype@gmail.com

${ }^{2}$ Medical College, Thrissur, Kerala, India 